\title{
Einfach mal anders gucken?!
}

\section{Neue Perspektiven auf alte Probleme: Sicherheitskultur in Industrie 4.0}

\author{
Anna Borg, Achim Buschmeyer, Claas Digmayer, Cornelia Hahn, \\ Eva-Maria Jakobs, Johanna Kluge, Jonathan Reinartz, Jan Westerbarkey \\ und Martina Ziefle
}

\subsection{Wer hat's gemacht: Die Projektpartner}

Die wesentliche Herausforderung in SiTra4.0 bestand in der systematischen Zusammenführung verschiedener fachlicher Perspektiven zu einem gemeinsamen Bezugsrahmen, der es erlaubt hat einen integrativen Zugang zu vernetzten Strukturen und daran gebundenen Qualifikationsanforderungen zu schaffen ${ }^{1}$. Die Ziele des Vorhabens waren nur interdisziplinär und unter Einbindung der praktischen Perspektive von Unternehmen erreichbar, entsprechend ist das Konsortium aufgestellt.

\footnotetext{
${ }^{1}$ Dieses Forschungs- und Entwicklungsprojekt wird durch das Bundesministerium für Bildung und Forschung (BMBF) im Programm „Innovationen für die Produktion, Dienstleistung und Arbeit von morgen“ (Förderkennzeichen 02L15A000- 02L15A004) gefördert und vom Projektträger Karlsruhe (PTKA) betreut. Die Verantwortung für den Inhalt dieser Veröffentlichung liegt bei den Autorinnen und Autoren.
}

\section{A. Borg $(\bowtie)$}

CBM Gesellschaft für Consulting Business und Management mbH,

Bexbach, Deutschland
A. Buschmeyer $\cdot$ C. Hahn
DERICHS u KONERTZ GmbH u Co KG, Aachen, Deutschland
C. Digmayer · E.-M. Jakobs
RWTH Aachen University, Textlinguistik und Technikkommunikation,
Human-Computer Interaction Center, Aachen, Deutschland

J. Kluge $\cdot$ M. Ziefle

RWTH Aachen University, Communication Science, Human-Computer Interaction Center, Aachen, Deutschland 
Westaflexwerk GmbH - Entwicklung und Erprobung einer Sicherheitskultur 4.0 für die Komponentenherstellung Westaflexwerk $\mathrm{GmbH}$ ist seit Generationen als Familien-Unternehmen in den Branchen Automobil, Haustechnik und Schienenfahrzeuge mit intelligenten Steuerungen und Dienstleistungen tätig. Mit eigenem Maschinen- und Werkzeugbau mittlerweile auch in additiven Geschäftsbereichen, wie der Elektromobilität und Wasserfiltration aktiv, stellt sich Westaflex dem digitalen Wandel und den unterschiedlichen Erwartungshaltungen von Kunden, Lieferanten und Mitarbeitern.

DERICHS u KONERTZ GmbH u Co. KG - Entwicklung und Erprobung einer Sicherheitskultur 4.0 für die Bauindustrie Seit 1926 betreut die DERICHS u KONERTZ Gruppe zuverlässig Projekte nach den stets gleichbleibenden Prinzipien: Professionalität, Effizienz und Innovation. Angefangen bei der Projektentwicklung über den schlüsselfertigen Bau bis hin zum Projektmanagement bildet DERICHS u KONERTZ den gesamten Immobilienzyklus aus einer Hand ab. Das Unternehmen sieht in der Digitalisierung die Schlüsseltechnologie für innovatives Planen und Bauen. Neben dem Einsatz von virtuellen Arbeitswerkzeugen zur Qualitäts- und Kostenkontrolle ist die Arbeit methodisch geprägt durch BIM (Building Information Modeling) und Lean Construction.

CBM Gesellschaft für Consulting Business und Management mbH - Entwicklung eines Kulturanalyse- und Gestaltungskonzeptes (Framework) für eine Sicherheitskultur 4.0 in KMU Die CBM ist in 2000 als Spin-off der RWTH Aachen University entstanden und betreibt Beratung, Umsetzung und Weiterbildung sowie Forschung und Entwicklung zu Arbeits-, Gesundheits- und Umweltschutz. Ein Schwerpunkt ist die Beratung von Unternehmen verschiedener Branchen und Größen zu Analyse von Sicherheitskultur und darauf aufbauend die Entwicklung einer Kultur der Prävention.

FIR e. V. an der RWTH Aachen - Entwicklung eines Transformationsprozesses für eine Sicherheitskultur 4.0 und dessen Implementierung Der FIR e. V. ist ein innovativ orientiertes Netzwerk mit über 200 Vertretern aus Forschung, Industrie und Verbänden. Das FIR forscht anwendungsorientiert unter dem Oberbegriff des Industrial Managements in den Bereichen der Betriebsorganisation und Unternehmensentwicklung. Ein besonderes Augenmerk liegt auf den am Markt verfügbaren Standard-IT-Lösungen.

\section{J. Reinartz}

Forschungsinstitut für Rationalisierung an der RWTH Aachen University,

Aachen, Deutschland

J. Westerbarkey

Westaflex GmbH, Gütersloh, Deutschland 
Human-Computer Interaction Center, RWTH Aachen University - Entwicklung verhaltensbeeinflussender Befähigungs- und Kommunikationskonzepte und Partizipationsmaßnahmen Das Human-Computer Interaction Center (HCIC) ist ein zentrales Institut der RWTH Aachen University, in dem eine interdisziplinäre Gruppe von Forschern sich aus unterschiedlichen Perspektiven mit den Themen MenschComputer-Interaktion, Usability, Risikokommunikation und Technologieakzeptanz beschäftigt. Das Zentrum betreibt akademische und von der Industrie finanzierte Forschung und Entwicklung. Im Projekt SiTra4.0 sind zwei Professuren des HCIC beteiligt: Die Professur Textlinguistik und Technikkommunikation (Leitung: Prof. Dr. phil. Eva-Maria Jakobs) sowie der Lehrstuhl für Communication Science (Leitung: Prof. Dr. phil. Martina Ziefle).

\subsection{Zielsetzung von SiTra4.0}

Ansätze zur Digitalisierung der Wirtschaft fokussieren meist technische Aspekte. SiTra4.0 erweitert diese Perspektive durch die Entwicklung eines Transformationsansatzes für die Etablierung eines präventiven, partizipativ erarbeiteten und gelebten Sicherheitskulturkonzeptes als maßgeblichen Erfolgsfaktor für die Umsetzung von Industrie 4.0 in KMU. Der Ansatz hilft Unternehmen, Ressourcen und Barrieren des Arbeitens in der digitalisierten Welt zu identifizieren, mit den Mitarbeitern ein passgenaues Sicherheitskulturkonzept $\mathrm{zu}$ entwickeln und dieses durch konkrete Maßnahmen in ihr Unternehmen einzubinden. Das Sicherheitskulturkonzept fokussiert Empowerment, Respekt und Vertrauen, Freiräume für Risiken sowie die Nutzung impliziten und expliziten Wissens der Mitarbeiter. Der Ansatz geht damit über bisherige Präventionsansätze hinaus. Er nutzt das Potenzial von Mitarbeitern und Führungskräften und setzt auf höhere Eigenverantwortung bei der Umsetzung von Industrie 4.0 in kleinen und mittleren Unternehmen (KMU).

Die Digitalisierung lässt die Grenzen zwischen Branchen und Unternehmen, aber auch jene innerhalb von Unternehmen fließend werden. An die Stelle der Grenzen treten Beziehungen, die situativ eingegangen und genauso schnell aufgehoben werden. Kaum erstaunlich, nimmt auch die Arbeitswelt die Gestalt eines Netzwerks an. Gemeint ist damit in erster Linie die Art und Weise, wie Menschen zusammenarbeiten und gemeinsam Wertschöpfung erbringen. Je anspruchsvoller die Aufgaben, desto bessere Hilfsmittel braucht es, um diese zu bewältigen. Die vierte industrielle Revolution ermöglicht mittels intelligenter und vernetzter Maschinen eben genau dieses. Menschen zeichnen sich dagegen gegenüber Maschinen gerade durch ihre vermeintlichen Schwächen aus. Sie sind irrational, verspielt, emotional und unberechenbar. Das macht uns Menschen kreativ und befähigt uns, Probleme oder Aufgaben mit kognitiver Leistungsfähigkeit zu bewältigen. Je mehr Maschinen es in einem Unternehmen gibt, desto gefragter sind eben diese menschlichen Fähigkeiten und desto komplexer und wichtiger wird die Mensch-Maschine Interaktion. 
Diese Schnittstellen im Unternehmen werden heutzutage noch viel zu wenig betrachtet. Gerade auf den verbesserten Umgang mit den Vorteilen aber auch den Nachteilen der neuen Technologien und den daraus resultierenden Belastungen wird zu wenig Aufmerksamkeit gelegt. Es fehlt ein ganzheitliches Sicherheitskulturkonzept, welches sich - im Sinne guter Arbeit - nicht nur mit den bekannten, sondern gerade auch mit den neuen sicherheitskritischen Themen beschäftigt. Eine Umsetzung einer „Sicherheitskultur 4.0“ kann nur abteilungs- und hierarchieübergreifend erreicht werden. Eine Sicherheitskultur die Mitarbeiter an sicheren und innovationsförderlichen Arbeitsaufgaben und -prozessen beteiligt mündet dabei auch in einem positiven Return on Invest.

In SiTra4.0 wurde ein Transformationsansatz entwickelt, der KMU auf dem Weg $\mathrm{zu}$ Industrie 4.0 bei der Entwicklung einer digitalen Sicherheitskultur mithilfe eines strukturierten Vorgehensmodells sowie geeigneter Strategien und Instrumente unterstützt. Hierdurch wird auch die meist eher technisch orientierten Herangehensweisen im Arbeits- und Gesundheitsschutz erweitert, indem ein partizipativ erarbeitetes Sicherheitskulturkonzept im Unternehmen implementiert wird. Damit hilft SiTra4.0 KMU, neue Anforderungen im AGS durch flexible Lösungen zu bewältigen, Ressourcen und Barrieren zu identifizieren, ein passgenaues Sicherheitskulturkonzept im Sinne eines ganzheitlichen AGS zu entwickeln und letztlich konkrete Maßnahmen zu erarbeiten. Dabei wirkt der in SiTra4.0 entwickelte Ansatz in zwei Richtungen: Einerseits werden Unternehmen, insbesondere KMU befähigt, die Herausforderung der Digitalisierung zu meistern, anderseits erweitert der Ansatz die klassischen Ansätze des AGS und der Sicherheitskultur.

\subsection{Entwicklung der Sicherheitskultur 4.0 in KMU}

Beschrieben werden wesentliche Implementierungsschritte in Form einer Roadmap, um eine Sicherheitskultur in kleinen und mittleren Betrieben $\mathrm{zu}$ entwickeln, die den Anforderungen und Herausforderungen der Digitalisierung und Industrie 4.0 gerecht werden. Das Vorgehen gliedert sich in sechs Schritte, die sukzessiv durchlaufen werden und am Bedarf - z. B. wenn neue Technologien im betreffenden Betrieb eingeführt oder neue Digitalisierungsrisiken identifiziert werden - adjustiert werden. So wird sichergestellt, dass der Sicherheitskulturansatz flexibel auf neue Entwicklungen reagieren kann. Um die Transformationsanforderungen ganzheitlich $\mathrm{zu}$ erfassen und $\mathrm{zu}$ beschreiben werden strukturiert sowohl die Prozesslandschaft und die unternehmerischen Abläufe in der Roadmap betrachtet als auch die verhaltenssteuernden Kulturaspekte integriert. Basis für die Integration des Kulturaspektes in den Transformationsansatz ist das in SiTra4.0 entwickelte Kulturindikatoren-Modell (s. Abb. 18.1 und [1]). Die Kulturindikatoren greifen die Aspekte des Arbeits- und Gesundheitsschutzes im Sinne einer Gestaltung von guter Arbeit im Rahmen der digitalen Transformation auf. Die relevanten Kulturindikatoren einer Sicherheitskultur 4.0 sind: Werte, Führung, Kommunikation, Einbindung und Regelung, die sich im Unternehmen auf 3 


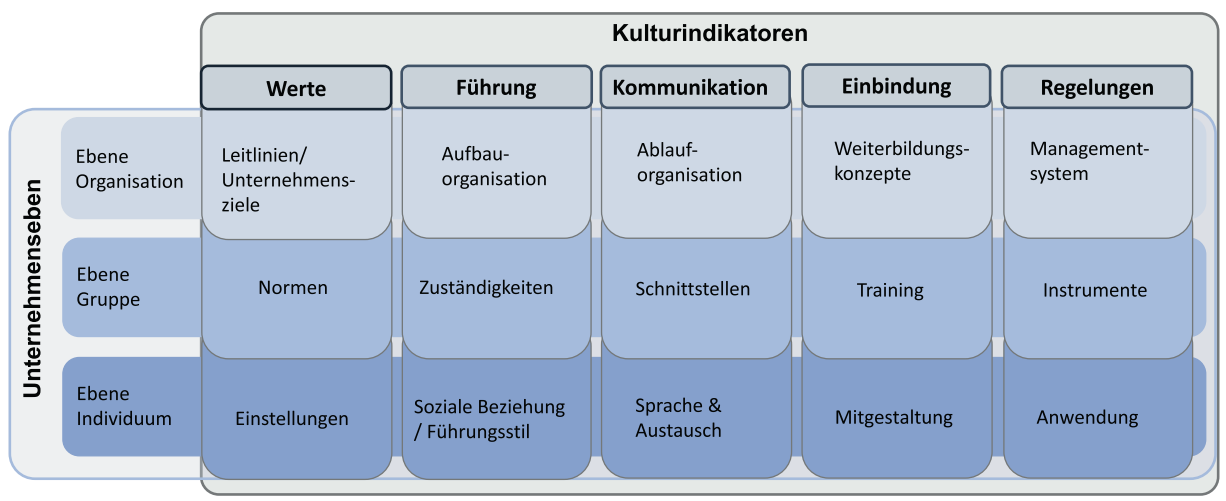

Abb. 18.1 SiTra4.0 Kulturindikatoren-Modell

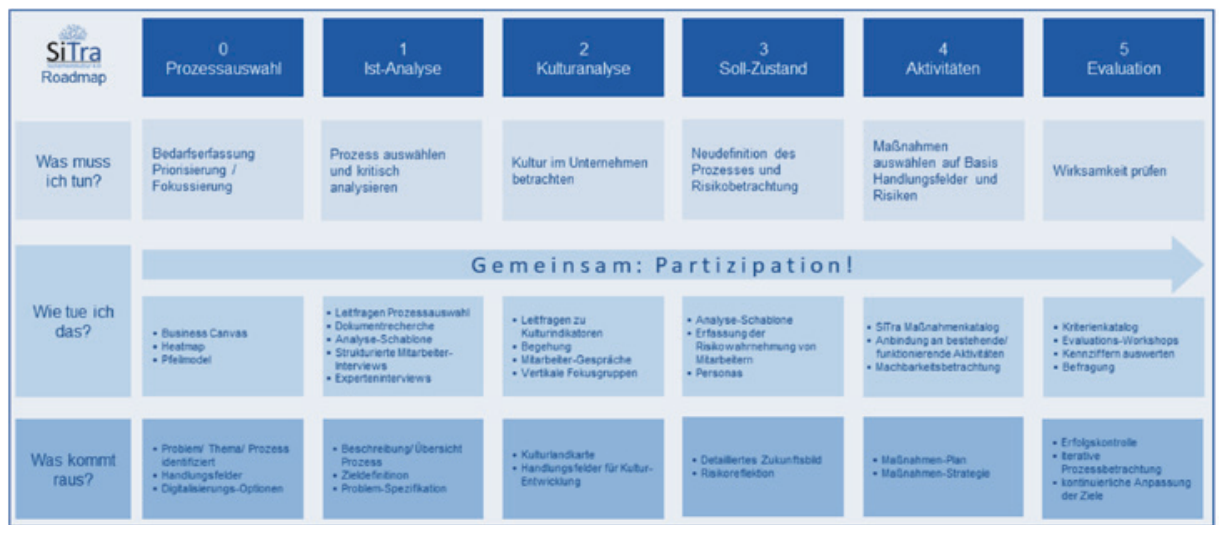

Abb. 18.2 SiTra4.0 Roadmap

Ebenen manifestieren: (1) der organisatorische Ebene, auf der Ziele, Strategie, Richtlinien und Regeln festgelegt werden, (2) der Gruppen-Ebene, auf der Regeln, Ziele und Richtlinien ausgearbeitet und in betrieblichen Handlungsanweisungen und Vorgehensweisen überführt werden und (3) der individuelle Ebene, auf der Ziele, Regeln und Richtlinien im Arbeitsalltag angewandt und in der ,betrieblichen Realität“ konkretisiert werden.

\subsubsection{Vorgehen: Die SiTra4.0-Roadmap}

Das Vorgehen anhand der Roadmap ist im Überblick in Abb. 18.2 dargestellt. Zu jedem Schritt (Phase 0 bis 5) werden das durchzuführende Vorgehen, die dafür nötigen Tools 
bzw. Methoden sowie die erwarteten Ergebnisse im Folgenden zusammenfassend dargestellt (ausführlichere Beschreibungen finden sich in [1]).

\section{Phase 0 (Prozessauswahl)}

Der erste Schritt der SiTra4.0-Roadmap initiiert die Bedarfserfassung sowie die Identifikation besonders kritischer Prozesse für den Transformationsprozess, umfasst jedoch noch keine konkreten Transformationsmaßnahmen, daher „Phase 0“. Zunächst gilt es ein allgemeines Verständnis über die wertschöpfenden Tätigkeiten des Unternehmens zu erhalten. Für diese Analyse bietet sich eine Kombination aus Interviews mit Führungskräften sowie die Anwendung des Business Model Canvas nach Osterwalder et al. ([2]) an, mit welchem das Geschäftsmodell visuell aufgenommen wird. Hierdurch werden eine gemeinsame Sprache und ein geteiltes Verständnis über die Wichtigkeit und Bestandteile der einzelnen Elemente generiert. Anhand des so identifizierten Wertschöpfungsprozesses des Unternehmens wird das entwickelte Heatmap Tool in Workshops mit Unternehmensvertretern befüllt (vgl. Abb. 18.3).

Ergebnis ist eine Bewertung der einzelnen Wertschöpfungsschritte auf den Ebenen Mensch-Maschinen, Technik und Organisation. Zur Identifikation des Digitalisierungspotenzials der einzelnen Prozesse werden in einer Vorschau - die detaillierte Erfassung erfolgt in Phase 1 - die Wertschöpfungsschritte im Detail mit Unternehmensvertretern hinsichtlich ihres Digitalisierungsgrades und dem daraus resultierenden Potenzial für die Digitalisierungsbausteine Business Intelligence, Cloud Computing, Mobile-Endgeräte sowie den zu betrachtenden Schnittstellen bewerten. Nach Abschluss der Phase 0 können anhand der vorliegenden Ergebnisse die Prozesse mit dem meisten Potenzial bzw. dem größten Handlungsbedarf für die weitere Bearbeitung identifiziert werden.

Phase 1 (Ist-Analyse, Problem-Analyse)

umfasst die Ist-Analyse der Digitalisierung der aktuellen Prozesslandschaft im Unternehmen. Hierfür wird ein Prozess aus Phase 0 ausgewählt und einer Prozessanalyse unterzogen. Für die Prozessanalyse bietet sich ein Methodenmix aus Interviews mit unternehmensinternen (z. B. Mitarbeitern, Managern) und unternehmensexternen Akteuren (z. B. Domänenexperten, Zulieferern) sowie einer Recherche von Unternehmensdokumenten an. Auf diese Weise soll eine möglichst umfassende Perspektive

\begin{tabular}{|c|c|c|c|c|c|c|}
\hline \multirow{2}{*}{$\begin{array}{l}\text { Sicherheitskultur } 4.0 \text { - } \\
\text { Handlungsbedarf }\end{array}$} & \multirow[b]{2}{*}{ Angebotsphase } & \multirow{2}{*}{$\begin{array}{l}\text { Bauvorbereitungs- } \\
\text { phase }\end{array}$} & \multicolumn{3}{|c|}{ Bauausführungsphase } & \multirow{2}{*}{$\begin{array}{l}\text { Gewährleistungs- } \\
\text { phase }\end{array}$} \\
\hline & & & $\begin{array}{l}\text { Überwachen der } \\
\text { Ausführung }\end{array}$ & $\begin{array}{c}\text { Baustellen- } \\
\text { bewirtschaftung }\end{array}$ & Controlling & \\
\hline $\begin{array}{c}\text { Benutzerschnittstelle } \\
\text { Mensch-Maschine-Schnittstelle }\end{array}$ & gering & mittel & hoch & mittel & hoch & gering \\
\hline $\begin{array}{l}\text { Soziotechnisches System } \\
\text { Technik und Organisation }\end{array}$ & gering & hoch & hoch & mittel & mittel & mittel \\
\hline $\begin{array}{c}\text { Organisation } \\
\text { Mensch und Organisation }\end{array}$ & gering & mittel & mittel & gering & mittel & mittel \\
\hline
\end{tabular}

Abb. 18.3 Heatmap zur Prozessauswahl 
auf den zu analysierenden Prozess gewonnen werden, die strukturiert erfasst wird. Bereits in diesem Schritt sollten aktiv die Mitarbeiter als Experten ihrer Arbeit und für die Abläufe im Unternehmen in die Analyse eingebunden werden, um die Akzeptanz gegenüber späterer Transformationsmaßnahmen zu gewährleisten. Mithilfe einer Schablone werden pro Prozessschritt der jeweilige Ist-Zustand, die (wichtigsten) Prozessbeteiligten, Schnittstellen zwischen Prozessbeteiligten, die im Schritt eingesetzten digitalen System sowie aktuelle Probleme erfasst. Die Erfassung schließt mit der Definition der Hauptziele zur Transformation dieses Prozesses. Wesentlicher Output von Phase 1 sind die Prozessbeschreibung, Zieldefinition und Problemspezifikationen der einzelnen Prozessschritte. Die Phase sollte für alle kritischen Prozesse in den aufgestellten Handlungsfeldern wiederholt werden.

\section{Phase 2 (Ist- und Soll-Analyse der Unternehmenskultur)}

fokussiert die aktuelle Unternehmenskultur (Ist-Analyse), definiert die gewünschte Kultur (Soll-Zustand) und leitet zu bearbeitende Handlungsfelder ab. Die Erfassung orientiert sich dabei an den fünf Kulturindikatoren: Werte, Führung, Kommunikation, Einbindung von Mitarbeitern und Regelungen und berücksichtigt sowohl die Organisations-, Gruppen- und Individuums-Ebene. Mittels Mitarbeitergesprächen, vertikaler Fokusgruppen, Workshops und Betriebsbegehungen soll ein möglichst umfassendes Bild der aktuellen Unternehmenskultur - Positiv- und Negativaspekte gewonnen werden. Um diese komplexe Aufgabe handhabbar zu machen, wird auf Basis des SiTra4.0-Kulturindikatoren-Modell (s. Abb. 18.1) eine Kulturlandkarte erstellt. Die Indikatoren werden bezogen auf den ausgewählten Digitalisierungsprozess erfasst und sowohl der Ist- Zustand wie auch der Zielzustand präzise beschrieben. Durch den Abgleich zwischen Ist und Ziel werden die Handlungsfelder für eine Transformation sichtbar und es können vorbereitend für Phase 4 entsprechend passgenaue Maßnahmen abgeleitet werden.

Das Vorgehen zur Erstellung der Kulturlandkarte orientiert sich an Leitfragen und baut sukzessive aufeinander auf. Zunächst wird übergeordnet - um eine erste Orientierung zur vorherrschenden Kultur im Unternehmen zu erheben - anhand von fünf Leitfragen (s. Tab. 18.1) ermittelt, wie sich der aktuelle Stand übergeordnet darstellt und ob der Kulturindikator für den ausgewählten Prozess eine relevante Stellgröße ist.

Im zweiten Schritt wird der in Phase 1 ausgewählte Prozess vertiefend betrachtet und bezüglich der Auswirkung auf die drei Unternehmensebenen differenziert (1) auf der organisatorisch/strategischen Ebene der Bezug zu den Unternehmenszielen, Strategien sowie Richtlinien (2) auf der Gruppen-Ebene der Bezug zu Gruppennormen und (3) auf der individuelle Ebene der Bezug zu individuellen Einstellung und Umsetzung im Arbeitsalltag. Wesentlich ist, dass hier nur die Bezüge und Auswirkungen Betrachtung finden, die für den Prozess Relevanz besitzen. Daraus ergibt sich eine passgenaue Abbildung der Unternehmenskultur, die unternehmensspezifische Voraussetzungen und Bedingungen berücksichtigt, wie z. B. branchenabhängige Prozesse und Prozessabläufe und der Einfluss der Digitalisierung. Ergebnis ist eine Abbildung der bestehenden Kultur 
Tab. 18.1 Leitfragen zu den Kulturindikatoren

\begin{tabular}{l|l}
\hline Kulturindikator & Leitfrage \\
\hline Werte & Welche Werte sind im Unternehmen verbreitet und gültig? \\
\hline Führung & Wie wird Führung im Unternehmen dargestellt und gelebt? \\
\hline Kommunikation & $\begin{array}{l}\text { Wie werden Informationen weitergeben? } \\
\text { Wie wird miteinander gesprochen? }\end{array}$ \\
\hline Einbindung & $\begin{array}{l}\text { Wie können sich Mitarbeitende aktiv einbringen? } \\
\text { Wird Weiterbildung am Bedarf angeboten? }\end{array}$ \\
\hline Regelungen & $\begin{array}{l}\text { Sind die Regeln im Unternehmen bekannt? } \\
\text { Wie werden die Regeln im Arbeitsalltag umgesetzt? }\end{array}$ \\
\hline
\end{tabular}

sowie der gewünschten Kultur in Form der Kulturlandkarte. Zusätzlich werden die relevanten Handlungsfelder für die Transformation und Kulturentwicklung sichtbar.

\section{Phase 3 (Soll-Zustand)}

dient zur Definition des Soll-Zustandes anhand einer Analyse-Schablone. Diese strukturiert die Neudefinition des Prozesses. Ausgefüllt werden hierbei Veränderungen bezogen auf die Schnittstellen, Akteure und digitale Systeme. Neu hinzu kommen die Definition des Soll-Zustandes und die Reflektion sich daraus ergebender zu erwartenden Risiken. Auf der Basis der im Projekt entstandenen empirischen Ergebnisse wurden Personas entwickelt, die in dieser Phase als Zusatzmaterial bereitgestellt werden. Die Personas dienen dazu, einen empirisch geleiteten Perspektivwechsel zu vollziehen und die Mitarbeiterperspektive in die Analyse möglicher wahrgenommener Risiken oder auch Nutzen zu integrieren.

\section{Phase 4 (Ableitung Aktionen)}

zielt auf die Auswahl durchzuführender Maßnahmen anhand der aufgestellten Handlungsfelder in Phase 2 und identifizierten Risiken in Phase 3. Die Auswahl erfolgt mittels eines im Projekt entwickelten Maßnahmenkatalogs, in dem einzelne Transformations-, Kommunikations- sowie verhaltensbeeinflussende Maßnahmen bezogen auf die Kulturindikatoren hinterlegt sind. Leitend für die Auswahl wird, der in Phase 1 ermittelte IstZustand in Hinblick auf zwei Fragestellungen betrachtet:

- Zu welchen kritischen Teilprozessen wurden noch keine Maßnahmen im Unternehmen eingeführt?

- $\mathrm{Zu}$ welchen kritischen Teilprozessen wurden Maßnahmen eingeführt, auf denen aufgebaut werden kann?

Dabei ist wichtig, dass Maßnahmen nicht nur punktuell eingesetzt werden sollten, vielmehr ist die Kombination verschiedener Maßnahmen zu einer ganzheitlichen 
Vorgehensstrategie erfolgsversprechend. So werden gezielt Lücken in der vorhandenen Maßnahmenlandschaft des Unternehmens (Fragestellung 1) und existierende Maßnahmen mit Optimierungspotenzial (Fragestellung 2) adressiert.

\section{Phase 5 (Evaluation)}

dient der Evaluation der durchgeführten Schritte. Grundlage dabei ist die in Phase 1 erarbeitete Zielvision. Die Überprüfung der Wirksamkeit der abgeleiteten Änderungen erfolgt zunächst auf der Basis des Abgleichs mit dieser Zielvision. Kernfragen sind dabei zunächst:

- Wurden die nötigen Änderungen an den in Phase 1 bis 3 identifizierten Prozessschritten erreicht?

- Konnte den in Phase 3 identifizierten Risiken entgegengewirkt bzw. diese aufgefangen werden?

- Sind für die Mitarbeiter negative Folgen des Transformationsprozesses aufgetreten, die nicht berücksichtigt wurden?

Als weitere Evaluationskriterien werden im Rahmen von SiTra4.0 zusätzliche Materialien erstellt, die vor allem die Integration der Mitarbeiterperspektive fokussieren. Im Rahmen des Projekts wurden auf der Basis quantitativer Erhebungen Evaluationsaspekte aus Arbeitnehmersicht untersucht. Dabei zeigten sich vor allem Aspekte wie eine gute Work-Life-Balance oder Arbeitnehmerzufriedenheit als relevant. Auf der anderen Seite sind Aspekte, die direkt mit der Bewältigung der Arbeitsaufgaben zusammenhängen, für die Arbeitnehmer von besonderer Bedeutung, wie die Erleichterung der Arbeit und gesteigerte Arbeitsleistung. Evaluationsmaße müssen also neben klassischen Kennziffern arbeitnehmer- und aufgabenspezifische Maße integrieren, um den Erfolg von einer durch das SiTra4.0 Vorgehensmodell geleiteten Transformation zu prüfen.

\subsection{Anwendung der Roadmap in der Praxis}

Das oben beschriebene Vorgehen wurde bei zwei Unternehmen aus den Branchen Bauindustrie und Metallbearbeitung exemplarisch umgesetzt. Die Implementierung wurde bei den Unternehmen DERICHS u KONERTZ und Westaflex im Projektverlauf nach der Systematik der SiTra4.0 Roadmap erprobt. Insgesamt wurde deutlich, dass beide Unternehmen von der in SiTra4.0 entwickelten methodischen und strukturierten Herangehensweise anhand der Roadmap profitieren, jedoch die Lösungen, Handlungsfelder und Umsetzungsstrategien branchenspezifisch differieren. Es zeigt sich, dass für eine Sicherheitskultur 4.0 und insbesondere für die notwendige Transformation und dem damit einhergehenden Kulturwandel es nicht die eine Herangehensweise gibt, sondern immer nur den unternehmensspezifischen Weg, der mithilfe der SiTra4.0 Kulturindikatoren abbildbar und bearbeitbar gemacht werden kann. 


\subsubsection{Umsetzung in der Bauindustrie}

Die Anwendung der Vorgehensweise gemäß der Roadmap für die Bauindustrie fand bei DERICHS u KONERTZ mit Fokus auf den Qualitätssicherungsprozesses auf der Baustelle statt. Im ersten Schritt (Phase 0) wurde analysiert, wo der dringendste Handlungsbedarf zur Digitalisierung unter Einbezug der Kulturaspekte vorhanden war. Im Ergebnis wurde auf die gesteuerte Qualitätskontrolle der Bauausführung fokussiert, da immer mehr Leistungen im Generalunternehmermodell an Subunternehmen weitergegeben werden. Aufgrund der heterogenen Subunternehmerstruktur und der daraus möglicherweise resultierender Ausführungsmängel kommt der Qualitätssicherung auf der Baustelle damit eine sehr hohe Bedeutung zu. An dieser Stelle bieten digitale Lösungen, die die Sicherheit in Bezug auf Kosten, Termine und Qualität erhöhen, ein besonderes Potenzial zur Verbesserung. Ergebnis war die Definition von Transformationszielen für den Prozess der Qualitätskontrolle auf der Baustelle.

In Phase 1 wurde nach der Festlegung des als besonders transformationsrelevanten Prozesses der Ist-Zustand systematisch erfasst. Wichtig war hier die Definition der Akteure, Schnittstellen und bereits verwendeten digitalen Systeme, außerdem die Analyse, welche Probleme es im Ist-Zustand gibt und welche Ziele sich daraus ableiten lassen. Ergebnis war, das im Prozess insbesondere die Schnittstelle zwischen der Projektvorbereitung und Bauausführung (interne Bauleitung und Poliere und externe Gewerke) im Fokus stehen muss. Hier kam es in der Vergangenheit immer wieder zu Informationsverlusten und großem Aufwand für eine - ohnehin meist unvollständige Dokumentation. Das Hauptziel war somit eine langfristige Qualitätskontrolle, die schon mit speziellen Prüfpunkten aus dem Wissen der Projektvorbereitung generiert werden soll. Zentral ist dabei, dass das Wissen von der Baustelle wieder zurück in die Planung bzw. Projektvorbereitung gespiegelt wird, wo es systematisch analysiert und ausgewertet werden kann. Wenn zum Beispiel eine Prüfung immer wieder fehlschlägt, sollte im nächsten Bauvorhaben dieser Punkt als systematischer Prüfpunkt verankern werden. Eine vollständige, digitalisierte Dokumentation von Mängeln und Beweissicherung von korrekt ausgeführter Leistung ist zudem für die spätere Gewährleistung von großer Bedeutung.

In Phase 2 (Ist- und Soll-Analyse der Unternehmenskultur) wurde die aktuelle Kultur anhand der Kulturindikatoren vor der Transformation bestimmt und die relevanten Themen hin zu einer Sicherheitskultur 4.0 skizziert. Dabei wurde zunächst unterschieden, welche Aspekte des Prozesses die Organisation, eine bestimmte Gruppe oder das Individuum von der Transformation betreffen. Im Beispiel des Qualitätssicherungsprozesses wurde festgestellt, dass in der Phase der Projektvorbereitung die ganze Gruppe von den Veränderungen durch die Digitalisierung betroffen sein wird. 
Als wesentliche Kulturindikatoren konnten dabei die Kommunikation und die Führung identifiziert werden. Bei der Bauausführung und beim Abschluss sind alle Bereiche (Organisation, Gruppe und Individuum) betroffen. Als zentrale Indikatoren der Sicherheitskultur4.0 konnten dabei die Kommunikation und die vorhandenen Regelungen identifiziert werden. Im Ergebnis konnte durch die Anwendung der SiTra4.0-Roadmap für den Prozess der Qualitätssicherung ein umfassendes Bild der maßgeblichen Kulturindikatoren erstellt werden.

In Phase 3 wurde aus den Transformationszielen der Phase 0 der SOLL-Zustand des Prozesses abgeleitet. Es wurden die Felder betrachtet, in denen es im neuen Prozess zu Veränderungen kommt. Bedingt durch die digitale Qualitätskontrolle, wird es zu einem Mehraufwand in der Projektvorbereitung kommen. Dem Mehraufwand gegenüber steht als klarer Vorteil, dass das Wissen aus allen Arbeitsschritten und von allen Projektbeteiligten in einer zentralen Datenbank zusammengeführt werden und für neue Bauvorhaben verwendet werden kann. Auch in der Gewährleistungsphase nach Bauabschluss können diese Informationen Anwendung finden. In der Bauausführung soll das System insbesondere die Mitarbeiter in der täglichen Arbeit unterstützen und das Risiko von Mängeln reduzieren. Beim Einsatz neuer digitaler Systeme in der Qualitätsprüfung konnten auch neue Risiken identifiziert werden. Einerseits besteht das Risiko, dass sich Mitarbeiter stärker überwacht fühlen und andererseits, dass sich die Mitarbeiter auf das System verlassen und Qualitätsmängel, die nicht explizit in den digitalen Prüflisten abgefragt werden, übersehen werden. Im Ergebnis konnte festgestellt werden, dass der Nutzen eines digitalen Qualitätssicherungssystems die Risiken überwiegt und durch eine Sicherheitskultur4.0 sinnvoll unterstützen wird.

In Phase 4 wurden bei DERICHS u KONERTZ aus der Bewertung der Phase 3 mit dem Hilfe des SiTra4.0-Maßnahmen-Kataloges die folgende Maßnahmen zur Gestaltung der Transformation hin zu einer Sicherheitskultur4.0 abgeleitet: Die Ausbildung von Key-Usern, regelmäßige Feedbackschleifen und spezielles Training und Schulungen für die Anwender des neuen digitalen Qualitätsprüfsystems.

Die Phase 5 (Evaluation) diente der Überprüfung der Wirksamkeit der Maßnahmen zur Umsetzung der abgeleiteten Veränderungen hin zu einer Sicherheitskultur4.0 bei DERICHS u KONERTZ. Dabei erfolgte der Abgleich des Transformationsfortschrittes mit der in Phase 0 entwickelten Zielvision. Im Ergebnis konnte für das Anwendungsbeispiel des Qualitätssicherungsprozesses auf der Baustelle festgestellt werden, dass die bisher erfolgten Umsetzungen bereits positive Effekte haben. Da sich die Umsetzung noch im Einführungsstadium befindet und insbesondere kulturelle Veränderungen im Unternehmen nicht kurzzeitig messbar sind, steht die langfristige Bewertung aus (s. Abschn. 18.5). 


\subsubsection{Umsetzung in der Komponentenherstellung der metallverarbeitenden Industrie}

Die Anwendung der Roadmap für die metallverarbeitende Industrie fand bei Westaflex anhand des Qualitätssicherungsprozesses in der Produktion statt. Im ersten Schritt (Phase 0) wurden die Bedarfe erfasst sowie besonders kritische Prozesse für den Transformationsprozess identifiziert. Anhand der Ergebnisse zeigten sich Digitalisierungspotenziale entlang der gesamten Wertschöpfungskette. Als besonders kritischer Prozess wurde die Qualitätskontrolle identifiziert und in den nachfolgenden Schritten der Roadmap bearbeitet. Die Qualitätskontrolle erstreckt sich von der Auftragsfreigabe durch den Kunden über die Arbeitsvorbereitung, die Auftragsbearbeitung durch die Baugruppenteams sowie die Kommissionierung bis hin zum Versand an den Kunden. Jeder Prozessschritt stellt unterschiedliche Anforderungen an die Qualitätskontrolle, bietet unterschiedliche Digitalisierungspotenziale und umfasst unterschiedliche Risiken - insbesondere an die Sicherheitskultur des Unternehmens.

In Phase 1 wurde der Ist-Zustand des betrachteten Prozesses erhoben in Hinsicht auf Akteure, Schnittstellen, digitale Systeme und aktuell bestehende Probleme. Es zeigt sich, dass in den meisten Schritten zwar digitale Systeme wie Enterprise Resource Planning (ERP), Barcodescanner oder Sensoren zur Erfassung von Maschinendaten eingesetzt werden. Jedoch werden Inhalte aus diesen Systemen derzeit nicht strategisch kombiniert und den Mitarbeitern digital entsprechend ihrer jeweiligen Rolle und Aufgaben zugänglich gemacht. Insbesondere Produktionsprozesse sind noch papierbasiert, was den Informationsstand, die Entscheidungsfreiheit und die Kommunikationsmöglichkeiten der Mitarbeiter - insbesondere in Sicherheitsfragen - einschränkt. Als Hauptziele für die Transformation wurden ERP als Steuerungssystem (für Projektplanung, Taktung und Dokumentation), Strukturierung der Kommunikation, eine mitlaufende Kalkulation sowie adäquate Assistenzsysteme für Mitarbeiter erarbeitet.

Phase 2 der SiTra4.0-Roadmap fokussierte bei einer Ist- und Soll-Analyse die Kulturaspekte der Transformation. Von Interesse war zunächst, ob Änderungen im betrachteten Prozess die gesamte Organisation, eine bestimmte Gruppe oder einzelne Individuen betreffen und welche der Kulturindikatoren in diesen Fällen besonders betroffen sind. Es zeigt sich, dass die Transformation auf Ebene der Organisation die Informationsflüsse sowie auf Ebene der Gruppe die Kommunikation an Schnittstellen zwischen Abteilungen stärken soll. Auf Ebene des Individuums sind insbesondere Möglichkeiten der Einbindung wichtig, die es erlauben, individuell wahrgenommene Optimierungsmöglichkeiten im Bereich der Sicherheit in den Prozess der Qualitätskontrolle einzubringen.

Im nächsten Schritt (Phase 3) wurde der Soll-Zustand des betrachteten Prozesses erarbeitet und Veränderungen bei einzelnen Prozessschritten erfasst. Als wesentlicher 
Punkt des Soll-Zustands wurde definiert, dass vorhandene Daten kein zweites Mal in die elektronischen Systeme eingeben werden sollen, sondern dass Mitarbeitern Kombinationen aus bestehenden Informationen unter einem bestimmten Bezugspunkt (z. B. Scanner-Barcode-Nummern) angeboten werden und in speziellen Assistenzsystemen (z. B. auf Tablets) dargestellt werden sollen. Auf diese Weise entstehen selbstentscheidende Teams, die einen vollständigen Informationsstand entsprechend Aufgaben und Rollen haben. Derartige Systeme sollen außerdem selbstlernend sein, z. B. indem wiederkehrende (Sicherheits-)Probleme oder Kundenvorgaben erfasst und beim wiederholten Aufrufen bestimmter Informationen automatisch mitangeboten werden, womit insgesamt die Qualitätskontrolle vereinfacht wird. Als wesentliches Risiko des SollZustandes wurde fehlende Akzeptanz der Mitarbeiter gegenüber den neueinzuführenden Systemen identifiziert.

Aufbauend auf den Erkenntnissen aus Phase 3 wurden in Phase 4 (Ableitung Aktionen) aus dem SiTra4.0-Maßnahmen-Katalog Ansätze zur Adressierung der identifizierten Risiken ausgewählt. Um Mitbestimmungsrechte und Akzeptanz gegenüber neuen digitalen Systemen von Beginn der Transformation an sicherzustellen, wurden einige Mitarbeiter als Key-User ausgewählt und sowohl in Workshops zur Gestaltung der Systeme als auch bei deren Evaluation vor Einführung integriert. Weitere Maßnahmen sollen Mitarbeiter in die Lage versetzen, mit den neu einzuführenden Systemen Sicherheitsprobleme in Form von Videotutorials zu erfassen und an Kollegen und Vorgesetzte zu kommunizieren. Die aus den Maßnahmen gewonnenen Erkenntnisse fließen in die Gestaltung von Schulungen für zukünftige System-Nutzer ein.

Phase 5 (Evaluation) ist zum Zeitpunkt der Fertigstellung dieser Publikation noch nicht abgeschlossen. Auf Mitarbeiterseite zeigen die Maßnahmen jedoch bereits Wirkung und erbrachten in Befragungen positives Feedback. Die im SiTra-Projekt begonnene Transformation soll über den Arbeitskreis „Sicherheit in einer Industrie 4.0“ (s. Abschn. 18.5) weiter begleitet werden.

\subsection{Forschungslücken und Ausblick}

Wie die Ergebnisse der beiden Anwendungsbeispiele verdeutlichen, vollzieht sich die kulturelle Transformation nicht in der gleichen Geschwindigkeit wie die technologische Entwicklung in Unternehmen. Gleichzeitig wird der Zustand einer ,fertigen“ und ,perfekten“ Sicherheitskultur nie abschließend erreicht werden können, denn eine resiliente Sicherheitskultur, die auf disruptive Innovationen und Risiken reagieren soll, bedarf des ständigen Monitorings sowie kontinuierlichem Streben nach Verbesserung. Aus diesen Gründen sollte der Erfolg der in SiTra4.0 entwickelten Roadmap und daran gebundener, durchgeführter Maßnahmen nicht auf kurze Sicht, sondern vielmehr 
langfristig evaluiert werden. Da eine solche Evaluationsphase über das Projektende hinausgeht, wurde im Projektkonsortium beschlossen, den SiTra4.0-Ansatz in einem Arbeitskreis für Sicherheit in Industrie 4.0 weiterzuführen und in diesem Rahmen die erzielten Ergebnisse und Methoden mit Praxispartnern zu erweitern (z. B. bezogen auf Branchenprofile) und zu verfeinern. Interessierte Unternehmen und Institutionen erhalten weitere Informationen auf der SiTra4.0-Website (https://sicherheitskultur40.de). Auf dieser Website wird ebenfalls in Kürze ein Demonstrator zu finden sein, der bei der Anwendung - im Sinne eines Leitfadens - die Umsetzung der SiTra4.0-Roadmap unterstützt.

\section{Projektpartner und Aufgaben}

- Westaflexwerk GmbH

Entwicklung und Erprobung einer Sicherheitskultur 4.0 für die Komponentenherstellung

- CBM Gesellschaft für Consulting Business und Management mbH Entwicklung eines Gestaltungsrahmens für eine Sicherheitskultur 4.0

- DERICHS u KONERTZ GmbH u Co. KG Entwicklung und Erprobung einer Sicherheitskultur 4.0 für die Bauindustrie

- RWTH Aachen University, Human-Computer Interaction Center, Professur Textlinguistik und Technikkommunikation (TLTK), Lehrstuhl für Communication Science (COMM)

Entwicklung eines Befähigungs- und Beteiligungskonzeptes für eine Sicherheitskultur

- FIR e. V. an der RWTH Aachen University

Entwicklung eines Transformationsprozesses für eine Sicherheitskultur 4.0 und dessen Implementierung

\section{Literatur}

1. Digmayer C, Jakobs E-M, Borg A, Buschmeyer A, Hahn C, Kluge J, Reinartz J, Westerbarkey J, Ziefle M (erscheint 2020, im Druck). Eine nachhaltige Sicherheitskultur als Transformationsansatz für Industrie 4.0 in kleinen und mittleren Unternehmen. In: Jeske T et al (Hrsg) Produktivitätsmanagement 4.0. Springer, Berlin

2. Osterwalder A et al (2011) Business model generation: a handbook for visionaries, game changers and challengers. Afr J Bus Manage 5(7):22-30 
Open Access Dieses Kapitel wird unter der Creative Commons Namensnennung 4.0 International Lizenz (http://creativecommons.org/licenses/by/4.0/deed.de) veröffentlicht, welche die Nutzung, Vervielfältigung, Bearbeitung, Verbreitung und Wiedergabe in jeglichem Medium und Format erlaubt, sofern Sie den/die ursprünglichen Autor(en) und die Quelle ordnungsgemäß nennen, einen Link zur Creative Commons Lizenz beifügen und angeben, ob Änderungen vorgenommen wurden.

Die in diesem Kapitel enthaltenen Bilder und sonstiges Drittmaterial unterliegen ebenfalls der genannten Creative Commons Lizenz, sofern sich aus der Abbildungslegende nichts anderes ergibt. Sofern das betreffende Material nicht unter der genannten Creative Commons Lizenz steht und die betreffende Handlung nicht nach gesetzlichen Vorschriften erlaubt ist, ist für die oben aufgeführten Weiterverwendungen des Materials die Einwilligung des jeweiligen Rechteinhabers einzuholen.

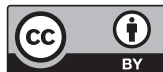

